\title{
RELATIVE CONTROLLABILITY OF NONLINEAR NEUTRAL VOLTERRA INTEGRODIFFERENTIAL SYSTEMS
}

\author{
K. BALACHANDRAN ${ }^{1}$ and J. P. DAUER ${ }^{2}$
}

(Received 21 June 1993)

\begin{abstract}
Sufficient conditions are derived for the relative controllability of nonlinear neutral Volterra integrodifferential systems with distributed delays in the control variables. The results are a generalization of previous results and are obtained by using Schauder's fixed-point theorem.
\end{abstract}

\section{Introduction}

The primary motivation for the study of neutral functional differential equations is the application to transmission-line theory. It is known that the mixed initial-boundary hyberbolic partial differential equation which arises in the study of lossless transmission lines can be replaced by an associated neutral differential equation. This equivalence has been the basis of a number of investigations of the stability properties of distributed networks (see [15]). In particular, models for systems with delay in the control occur in population studies and in some complex economic systems. More specifically, models for systems with distributed delays in the control occur in the study of agricultural economics and population dynamics $[2,3]$. Volterra integrodifferential equations occur often in applied mathematics [7]. In [12] a simplified model for compartmental systems with pipes is represented by nonlinear neutral Volterra integrodifferential equation.

The problem of controllability of linear neutral systems has been investigated by several authors [6, 13, 5]. Angell [1] and Chukwu [8] discussed the functional controllability of nonlinear neutral systems and Underwood and Chukwu [17] studied the null controllability for such systems. Further Chukwu [9] considered the Euclidean

\footnotetext{
'Department of Mathematics, Bharathiar University, Coimbatore-641 046, TAMIL NADU, INDIA.

${ }^{2}$ Department of Mathematics, University of Tennessee at Chattanooga, Chattanooga, Tennessee 37415, USA.

(C) Australian Mathematical Society, 1996, Serial-fee code 0334-2700/95
} 
controllability of a neutral system with nonlinear base. Onwuatu [16] discussed the problem for nonlinear systems of neutral functional differential equations with limited controls. Gahl [11] derived a set of sufficient conditions for the controllability of nonlinear neutral systems through the fixed-point method. In [4] Balachandran established sufficient conditions for the controllability of nonlinear neutral Volterra integrodifferential systems. However for systems with delays in the control variables the problem of relative controllability is still to be studied. A number of papers have appeared on linear systems with different types of delays in control variables. Klamka [14] and Balachandran and Dauer [5] investigated the relative controllability of nonlinear systems with distributed delays in control. In this paper we shall study the relative controllability of nonlinear neutral Volterra integrodifferential systems with distributed delays in control. Our approach, similar to one used by Do [10] for nonlinear neutral systems, is to define the appropriate control and its corresponding solution by an integral equation. This equation is then solved by applying the Schauder fixed-point theorem.

\section{Preliminaries}

Let $Q$ denote the Banach space of continuous $R^{n} \times R^{m}$-valued functions defined on $\left[0, t_{1}\right]$ with the norm $\|(x, u)\|=\|x\|+\|u\|$, where $\|x\|=\sup \left\{|x(t)|, t \in\left[0, t_{1}\right]\right\}$ and $\|u\|=\sup \left\{|u(t)|, t \in\left[0, t_{1}\right]\right\}$.

That is, $Q=C_{n}\left[0, t_{1}\right] \times C_{m}\left[0, t_{1}\right]$, where $C_{n}\left[0, t_{1}\right]$ is the Banach space of continuous $R^{n}$-valued functions defined on $\left[0, t_{1}\right]$ with the supremum norm. Put $J=\left[0, t_{1}\right]$.

Consider the linear neutral Volterra integrodifferential systems with distributed delays of the form

$$
\begin{aligned}
\frac{d}{d t}[ & \left.x(t)-\int_{0}^{t} C(t, s) x(s) d s-g(t)\right] \\
& =A(t) x(t)+\int_{0}^{t} G(t, s) x(s) d s+\int_{-h}^{0} d_{\theta} B(t, \theta) u(t+\theta)
\end{aligned}
$$

and the nonlinear system

$$
\begin{aligned}
& \frac{d}{d t}\left[x(t)-\int_{0}^{t} C(t, s) x(s) d s-g(t)\right] \\
& \quad=A(t) x(t)+\int_{0}^{t} G(t, s) x(s) d s+\int_{-h}^{0} d_{\theta} B(t, \theta) u(t+\theta)+f(t, x(t), u(t)) .
\end{aligned}
$$

Here $x \in R^{n}$ and $u$ is an $m$-dimensional vector function with $u \in C_{m}\left[-h, t_{1}\right]$ and $B(t, \theta)$ is an $n \times m$ matrix continuous in $t$ and of bounded variation in $\theta$ on $[-h, 0]$ 
for each $t \in J$. The $n \times n$ matrices $A(t), C(t, s)$ and $G(t, s)$ are continuous in their arguments. The $n$-vector functions $f$ and $g$ are respectively continuous and absolutely continuous. The integral is in the Lebesgue-Stieltjes sense which is denoted by the symbol $d_{\theta}$.

Let $h>0$ be given. For a function $u:\left[-h, t_{1}\right] \rightarrow R^{m}$ and $t \in\left[0, t_{1}\right]$ we use the symbol $u_{t}$ to denote the function on $[-h, 0)$ defined by $u_{t}(s)=u(t+s)$ for $s \in[-h, 0)$. The following definitions of complete state and relative controllability of system (1) or (2) are assumed [14].

DEFINITION 1. The set $z(t)=\left\{x(t), u_{t}\right\}$ is said to be the complete state of the system (1) at time $t$.

DEFINITION 2. The system (1) or (2) is said to be relatively controllable on $J$ if, for every initial complete state $z(0)$ and $x_{1} \in R^{n}$, there exists a control function $u(t)$ defined on $\left[0, t_{1}\right]$ such that the solution of the system (1) or (2) satisfies $x\left(t_{1}\right)=x_{1}$.

The solution of (1) can be written as in [18]:

$$
\begin{gathered}
x(t)=Z(t, 0)[x(0)-g(0)]+g(t)-\int_{0}^{t}(\partial / \partial t) Z(t, s) g(s) d s \\
+\int_{0}^{t} Z(t, s)\left(\int_{-h}^{0} d_{\theta} B(s, \theta) u(s+\theta)\right) d s,
\end{gathered}
$$

where $Z(t, s)$ and $(\partial / \partial t) Z(t, s)$ are continuous matrices satisfying

$$
\begin{gathered}
(\partial / \partial t) Z(t, s)-\int_{0}^{t}(\partial / \partial t) Z(t, \tau) C(\tau, s) d \tau+C(t, s) \\
=-Z(t, s) A(s)-\int_{0}^{t} Z(t, \tau) G(\tau, s) d \tau,
\end{gathered}
$$

and where $Z(t, t)=I$ and the solution of the nonlinear system (2) is given by

$$
\begin{gathered}
x(t)=Z(t, 0)[x(0)-g(0)]+g(t)-\int_{0}^{t}(\partial / \partial t) Z(t, s) g(s) d s \\
+\int_{0}^{t} Z(t, s)\left[\int_{-h}^{0} d_{\theta} B(s, \theta) u(s+\theta)+f(s, x(s), u(s)] d s .\right.
\end{gathered}
$$

Using the asymmetric Fubini theorem, as in [14], equation (3) can be written as

$$
\begin{aligned}
x(t) & =Z(t, 0)[x(0)-g(0)]+g(t)-\int_{0}^{t}(\partial / \partial t) Z(t, s) g(s) d s \\
& +\int_{-h}^{t} d_{B_{\theta}} \int_{\theta}^{0} Z(t, s-\theta) B(s-\theta, \theta) u_{\circ}(s) d s \\
& +\int_{0}^{t}\left[\int_{-h}^{0} Z(t, s-\theta) d_{\theta} B_{t}(s-\theta, \theta)\right] u(s) d s,
\end{aligned}
$$


where $d_{B_{\theta}}$ denotes that the integration is in the Lebesgue-Stieltjes sense with respect to the variable $\theta$ in $B$ and

$$
B_{\imath}(s, \theta)= \begin{cases}B(s, \theta), & s \leq t \\ 0 & s>t\end{cases}
$$

Define

$$
\begin{gathered}
p(t)=Z(t, 0)[x(0)-g(0)]+g(t)-\int_{0}^{t}(\partial / \partial t) Z(t, s) g(s) d s, \\
q(t)=\int_{-h}^{0} d_{B_{\theta}} \int_{\theta}^{0} Z(t, s-\theta) B(s-\theta, \theta) u_{\circ}(s) d s, \\
S(t, s)=\int_{-h}^{0} Z(t, s-\theta) d_{\theta} B_{t}(s-\theta, \theta)
\end{gathered}
$$

and the controllability matrix

$$
W(0, t)=\int_{0}^{t} S(t, s) S^{*}(t, s) d s,
$$

where the star $\left(^{*}\right)$ denotes the matrix transpose.

Then equations (5) and (4) become

$$
x(t)=p(t)+q(t)+\int_{0}^{t} S(t, s) u(s) d s
$$

and

$$
x(t)=p(t)+q(t)+\int_{0}^{t} S(t, s) u(s) d s+\int_{0}^{t} Z(t, s) f(s, x(s), u(s)) d s .
$$

It is easy to prove that, as in [4], the system (1) is relatively controllable on $J$ if and only if $W$ is nonsingular.

It is clear that $x_{1}$ can be obtained if there exist continuous $x$ and $u$ such that

$$
u(t)=S^{*}\left(t_{1}, t\right) W^{-1}\left(0, t_{1}\right)\left[x_{1}-p\left(t_{1}\right)-q\left(t_{1}\right)-\int_{0}^{t_{1}} Z\left(t_{1}, s\right) f(s, x(s), u(s)) d s\right]
$$

and

$$
x(t)=p(t)+q(t)+\int_{0}^{t} S(t, s) u(s) d s+\int_{0}^{t} Z(t, s) f(s, x(s), u(s)) d s .
$$


Now we must find the conditions for the existence of such $x$ and $u$. If $\alpha_{i} \in L^{1}(J)$, $i=1,2, \ldots q$, the $\left\|\alpha_{i}\right\|$ is the $L^{1}$ norm of $\alpha_{i}(s)$. That is, $\left\|\alpha_{i}\right\|=\int_{0}^{t}\left|\alpha_{i}\right| d s$. Let us introduce the notation

$$
\begin{aligned}
K & =\max \left\{\|Z(t, s)\|: 0 \leq s \leq t \leq t_{1}\right\}, \\
k & =\max _{0 \leq s \leq t_{1}}\left\{S\left(t_{1}, s\right) t_{1}, 1\right\}, \\
a_{i} & =3 k \max _{0 \leq t \leq t_{1}}\left\{\left\|S^{*}\left(t_{1}, t\right)\right\| \| W^{-1}\left(0, t_{1}\|\| Z\left(t_{1}, t\right)\|\| \alpha_{i} \|\right\},\right. \\
b_{i} & =3 K\left\|\alpha_{i}\right\|, \\
c_{i} & =\max \left\{a_{i}, b_{i}\right\}, \\
d_{1} & =3 k \max _{0 \leq t \leq t_{1}}\left\|S^{*}\left(t_{1}, t\right)\right\| \| W^{-1}\left(0, t_{1} \|\left[\left|x_{1}\right|+\mid p\left(t_{1}|+| q_{1} \mid\right],\right.\right. \\
d_{2} & =3\left[\left|p\left(t_{1}\right)\right|+\left|q\left(t_{1}\right)\right|\right], \\
d & =\max \left\{d_{1}, d_{2}\right\} .
\end{aligned}
$$

\section{Main result}

Now we shall prove the main theorem which is a generalization of Theorem 2 in [4].

THEOREM. Let measurable functions $\phi_{i}: R^{n+m} \rightarrow R^{+}$and $L^{1}$ functions $\alpha_{i}: J \rightarrow R^{+}$, $i=1,2, \ldots q$ be such that

$$
|f(t, x, u)| \leq \sum_{i=1}^{q} \alpha_{i}(t) \phi_{i}(x, u) \quad \text { for every } \quad(t, x, u) \in J \times R^{n+m} .
$$

Then the relative controllability of (1) implies the relative controllability of (2) if

$$
\lim _{r \rightarrow \infty} \sup \left(r-\sum_{i=1}^{q} c_{i} \sup \left\{\phi_{i}(x, u):\|(x, u)\| \leq r\right\}\right)=\infty .
$$

PROOF.

$$
\begin{gathered}
\text { Define } T: Q \rightarrow Q \text { by } \\
T(x, u)=(y, v),
\end{gathered}
$$

where

$$
v(t)=S^{*}\left(t_{1}, t\right) W^{-1}\left(0, t_{1}\right)\left[x_{1}-p\left(t_{1}\right)-q\left(t_{1}\right)-\int_{0}^{t_{1}} Z\left(t_{1}, s\right) f(s, x(s), u(s)) d s\right]
$$


and

$$
y(t)=p(t)+q(t)+\int_{0}^{t} S(t, s) v(s) d s+\int_{0}^{t} Z(t, s) f(s, x(s), u(s)) d s .
$$

By our assumptions, the operator $T$ is continuous. Clearly the solution $u$ and $x$ to (8) and (9) are fixed points of $T$. We shall prove the existence of such fixed points by using the Schauder fixed-point theorem.

Let $\Psi_{i}(r)=\sup \left\{\phi_{i}(x, u):\|(x, u)\| \leq r\right\}$. Since (10) holds, there exists $r_{\circ}>0$ such that

$$
\sum_{i=1}^{q} c_{i} \Psi_{i}\left(r_{\mathrm{o}}\right)+d \leq r_{\mathrm{o}}
$$

Now let

$$
Q_{r_{\mathrm{o}}}=\left\{(x, u) \in Q:\|(x, u)\| \leq r_{\mathrm{o}}\right\} .
$$

If $(x, u) \in Q_{r_{\mathrm{o}}}$ then from (11) and (12) we have

$$
\begin{aligned}
\|v\| \leq & \left\|S^{*}\left(t_{1}, t\right)\right\|\left\|W^{-1}\left(0, t_{1}\right)\right\|\left[\left|x_{1}\right|+\left|p\left(t_{1}\right)\right|+\left|q\left(t_{1}\right)\right|\right. \\
& \left.\quad+\int_{0}^{t_{1}}\left\|Z\left(t_{1}, s\right)\right\| \sum_{i=1}^{q} \alpha_{i}(s) \phi_{i}(x(s), u(s)) d s\right] \\
& \leq\left(d_{1} / 3 k\right)+(1 / 3 k) \sum_{i=1}^{q} \alpha_{i} \Psi_{i}\left(r_{0}\right) \\
& \leq(1 / 3 k)\left(d+\sum_{i=1}^{q} c_{i} \Psi_{i}\left(r_{0}\right)\right) \\
& \leq\left(r_{\circ} / 3 k\right) \leq\left(r_{\circ} / 3\right)
\end{aligned}
$$

and

$$
\begin{aligned}
\|y\| & \leq|p(t)|+|q(t)|+\int_{0}^{t}\|S(t, s)\|\|v\| d s+\int_{0}^{t}\|Z(t, s)\| \sum_{i=1}^{q} \alpha_{i} \phi_{i}(x(s), u(s)) d s \\
& \leq(d / 3)+k\|v\|+K \sum_{i=1}^{q}\left\|\alpha_{i}\right\| \Psi_{i}\left(r_{\mathrm{o}}\right) \\
& \leq(d / 3)+k\|v\|+1 / 3 \sum_{i=1}^{q} c_{i} \Psi_{i}\left(r_{\mathrm{o}}\right) \\
& \leq(1 / 3)\left(d+\sum_{i=1}^{q} c_{i} \Psi_{i}\left(r_{\mathrm{o}}\right)\right)+k\|v\| \\
& \leq\left(r_{\mathrm{o}} / 3\right)+\left(r_{\mathrm{o}} / 3\right)=2\left(r_{\mathrm{o}} / 3\right) .
\end{aligned}
$$


Hence $T$ maps $Q_{r_{\mathrm{o}}}$ into itself. Further it is easy to see that $T\left(Q_{r}\right)$ is equicontinuous for all $r>0$ [10]. By the Ascoli-Arzela theorem, $T\left(Q_{r_{0}}\right)$ is compact in $Q$. Since $Q_{r_{\mathrm{o}}}$ is closed, bounded and convex, the Schauder fixed-point theorem guarantees that $T$ has a fixed point $(x, u) \in Q_{r_{0}}$ such that $T(x, u)=(x, u)$. It follows that, for $(x, u) \equiv(y, v)$, we have

$$
x(t)=p(t)+q(t)+\int_{0}^{t} S(t, s) u(s) d s+\int_{0}^{t} Z(t, s) f(s, x(s), u(s)) d s .
$$

Thus the solutions of (8) and (9) exist. Hence the system is relatively controllable on $J$.

REMARK. To apply the above theorem we must construct $\alpha_{i}$ s and $\phi_{i}$ s such that (10) is satisfied. These constructions are different for different situations. However an obvious construction of $\alpha_{i}$ s and $\phi_{i}$ s is easily achieved by taking $q=1, \alpha_{1}=\alpha=1$ and

$$
\phi_{1}(x, u)=\phi(x, u)=\sup \{|f(t, x, u)|: t \in J\} .
$$

In this case (10) holds if

$$
\lim _{r \rightarrow \infty} \inf (1 / r) \sup \{\phi(x, u):\|(x, u)\| \leq r\}<1 / c_{1} .
$$

Now we state a corollary which is a particular case of the above theorem.

COROLLARY. If the continuous function $f$ satisfies the condition

$$
\lim _{\|(x, u)\| \rightarrow \infty}|f(t, x, u)| /\|(x, u)\|
$$

uniformly in $t \in J$ and if the system (1) is relatively controllable on $J$, then the system (2) is relatively controllable on $J$.

\section{Acknowledgement}

A part of the work was done while the first author was visiting the University of Tennessee at Chattanooga during May 1993, and he is thankful to Professor J. P. Dauer for arranging this visit. Also the work was partly supported by CSIR, New Delhi.

\section{References}

[1] T. S. Angell, "On controllability for nonlinear hereditary systems; A fixed point approach", Nonlinear Analysis, Theory, Methods and Applications 4 (1990) 529-548. 
[2] Z. Artstein, "Linear systems with delayed controls-A reduction", IEEE Transactions on Automatic Control AC-27 (1982) 869-879.

[3] Z. Artstein and G. Tadmor, "Linear systems with indirect controls - The underlying measures", SIAM Journal on Control and Optimization 20 (1982) 96-111.

[4] K. Balachandran, "Controllability of neutral Volterra integrodifferential systems", Journal of Australian Mathematical Society, Ser. B 34 (1992) 18-25.

[5] K. Balachandran and J. P. Dauer, "Relative controllability of perturbations of nonlinear systems", Journal of Optimization Theory and Applications 63 (1989) 51-56.

[6] H. T. Banks and G. A. Kent, "Control of functional differential equations of retarded and neutral type to target sets in function space", SIAM Journal on Control 10 (1972) 562-593.

[7] T. A. Burton, Volterra Integral and Differential Equations (Academic Press, New York, 1983).

[8] E. N. Chukwu, "Functional inclusion and controllability of nonlinear neutral functional differential systems", Journal of Optimization Theory and Applications 29 (1979) 291-300.

[9] E. N. Chukwu, "On the Euclidean controllability of a neutral system with nonlinear base", Nonlinear Analysis, Theory, Methods and Applications 11 (1987) 115-123.

[10] V.N. Do, "Controllability of semilinear systems", Journal of Optimization Theory and Applications 65 (1990) 41-52.

[11] R. D. Gahl, "Controllability of nonlinear systems of neutral type", Journal of Mathematical Analysis and Applications 63 (1978) 33-42.

[12] I. Gyori and J. Wu, "A neutral equation arising from compartmental systems with pipes", Journal of Dynamics and Differential Equations 3 (1991) 289-311.

[13] M. Q. Jacobs and C. E. Langenhop, "Criteria for function space controllability of linear neutral systems", SIAM Journal on Control and Optimization 14 (1976) 1009-1048.

[14] J. Klamka, "Relative controllability of nonlinear systems with distributed delays in control", International Journal of Control 28 (1978) 307-312.

[15] D. A. O'Conner, "State controllability and observability for linear neutral systems", D.Sc. Thesis, Washington University, 1978.

[16] J. U. Onwuatu, "On the null controllability in function space of nonlinear systems of neutral functional differential equations with limited controls", Journal of Optimization Theory and Applications 42 (1984) 397-420.

[17] R. G. Underwood and E. N. Chukwu, "Null controllability of nonlinear neutral differential equations", Journal of Mathematical Analysis and Applications 129 (1988) 326-345.

[18] J. Wu, "Periodic solutions of nonconvolution neutral integrodifferential equations", Acta Math. Sci 8 (1988) 307-314. 